\title{
Performance Assessment of the Quality-Oriented Adaptation Scheme
}

\author{
Gabriel-Miro Muntean ${ }^{1}$, Philip Perry ${ }^{1}$, and Liam Murphy ${ }^{2}$ \\ ${ }^{1}$ School of Electronic Engineering, Dublin City University, \\ Glasnevin, Dublin 9, Ireland \\ \{munteang, perryp\}@eeng.dcu.ie \\ http://www. eeng.dcu.ie/ munteang \\ ${ }^{2}$ Computer Science Department, University College Dublin, \\ Belfield, Dublin 4, Ireland \\ liam.murphy@ucd.ie
}

\begin{abstract}
This paper focuses on the experimental performance assessment of the Quality-Oriented Adaptation Scheme (QOAS) when used for streaming high quality multimedia-based services via local broadband IP networks. Results of objective tests using a QOAS simulation model show very efficient adaptation in terms of end-user perceived quality, loss rate, and bandwidth utilization, compared to existing adaptive streaming schemes such as LDA+ and TFRCP. Subjective tests confirm these results by showing high end-user perceived quality of the QOAS under various network conditions.
\end{abstract}

\section{Introduction}

Bursty losses, or excessive and extremely variable delays, caused by increased traffic have a devastating effect on multimedia delivery over IP networks by severely affecting the end-users' perceived quality. Regardless of the infrastructure architecture used for delivering rich content multimedia-based services [1], the service providers and network operators aim at increasing its utilization and thus their revenues. On the other hand the customers always want the best quality for the services at the lowest price possible.

The Quality-Oriented Adaptation Scheme (QOAS) - an end-to-end applicationlevel adaptive control solution proposed in [2], [3] and described in [4], [5], [6] balances these opposing requirements and works best in increased traffic conditions. The adaptation is based on a client-located grading scheme that maps some networkrelated parameters' values and variations to application-level scores that describe the quality of delivery. In order to maximize the quality of service in existing conditions, estimates of end-user perceived quality are actively considered during this grading process. The computed quality scores are used by a server-side feedback-controlled QOAS mechanism to take adaptive decisions.

Results of extensive testing that assess QOAS in local broadband IP-networks [7] are presented and discussed in this paper. They illustrate QOAS performance and its potential benefits for delivering multimedia-based services to the customers. These tests involve both simulations and subjective perceptual testing and their results are 
presented in section 4. Section 2 discusses some related work whereas section 3 gives details about QOAS. At the end of the paper, performance analysis, conclusions and possible future work directions are presented.

\section{Related Work}

Extensive research has focused on proposing different adaptive schemes based on rate control and various directions have been taken. They were mainly classified in the literature [8], [9], [10] according to the place where the adaptive decision is taken.

Source-based adaptive control techniques require the sender to respond to variations in the delivery conditions. Among them there are solutions based on probing tests that try to estimate the available bandwidth while maintaining the loss rate below a certain threshold [11], [12].

Other solutions follow a throughput model that determines the transmission rate in certain conditions. The TCP-Friendly Rate Control Protocol (TFRCP)-based adaptive scheme [13] relies only on the TCP model proposed in [14], whereas the Loss-Delay Adjustment Algorithm (LDA) [15] also uses another model for rate adaptation.

A third direction that relies on heuristic knowledge, experimental testing and models encompasses many of the proposed schemes. Among the most significant are the Loss-Delay-based Adaptation Algorithm (LDA+) [16] that extends LDA; the Rate Adaptation Protocol (RAP) [17] which uses a similar approach to TCP's AIMD adaptation; Layered Quality Adaptation (LQA) [18] that bases its rate control on a layered approach; and the scheme described in [19] that bases its adaptation on information about the network state acquired by a TCP-like mechanism.

Receiver-based schemes provide mechanisms that allow for the receivers to select the service quality and/or rate, such as Receiver-driven Layered Multicast (RLM) [20] and Receiver-driven Layered Congestion Control (RLC) [21]. Among the hybrid adaptive mechanisms that involve both the sender and the receiver in the adaptation process, the TCP Emulation At Receivers (TEAR) scheme was described in detail in [22]. Transcoder-based solutions focus on matching the available bandwidth of heterogeneous receivers through transcoding or filtering, and significant solutions were presented in [23], [24].

Commercial adaptive streaming solutions like Real Networks' SureStream [25] and Microsoft's Multimedia Multi-bitrate (MBR) solution [26] are proprietary and detailed technical information has never been revealed. However the available information states that they were specially designed to allow for adaptations at very low bit-rates, unlike QOAS which addresses high quality high bit-rate video streaming.

\section{Quality-Oriented Adaptation Scheme (QOAS)}

Although the adaptive schemes presented in the "Related Work" section have shown good adaptation results in certain scenarios, their adjustment policies are not directly related to the quality of the streaming process as perceived by the customers. Unlike them, QOAS bases its adaptation process on estimates of the end-user perceived qual- 
ity made at the receiver. This perceived quality is estimated in-service using the noreference moving picture quality metric-Q proposed in [27] that describes the joint impact of MPEG rate and data loss on video quality. More details about $\mathrm{Q}$ and its usage are presented in [4].

QOAS is distributed and consists of server-side and client-side components. It makes use of a client-located Quality of Delivery Grading Scheme (QoDGS) and of a Server Arbitration Scheme (SAS) that co-operate in order to implement the feedbackcontrolled adaptation mechanism. The QOAS principle is schematically illustrated in Figure 1 for pre-recorded multimedia streaming used for Video-on-Demand (VoD) services, and is briefly described next.

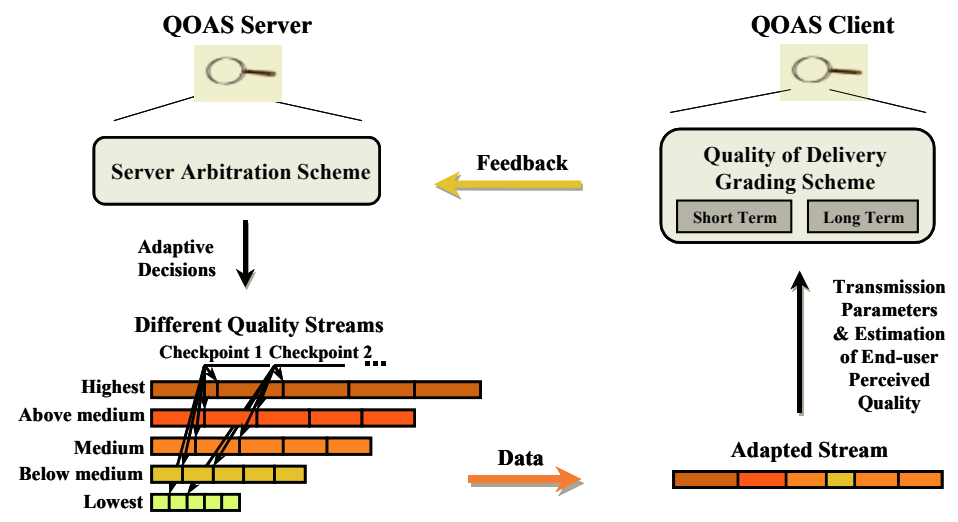

Fig. 1. QOAS principle, illustrated for pre-recorded multimedia streaming.

\subsection{Principle of Quality-Oriented Adaptive Scheme}

Multimedia data is received at the client where the QoDGS continuously monitors both some network-related parameters such as loss rate, delay and jitter and the estimated end-user perceived quality. According to their values and variations, QoDGS grades the quality of delivery (QoD) in terms of application-level quality scores $\left(\mathrm{QoD}_{\text {Scores }}\right)$ that are sent to the server as feedback. These scores are analyzed by the SAS that may suggest taking adaptive decisions in order to maximize the end-user perceived quality in existing delivery conditions. These decisions affect an internal state defined for the QOAS server component that was associated with the streamed multimedia clip's quality as shown in Figure 1. The figure presents the five-state quality model used during testing with the following states: excellent, good, average, poor and bad. Between adjacent states the adaptation step is $0.5 \mathrm{Mbps}$ in the experiments described in this paper. Any QOAS server state modification affects the multimedia data transmission rate. For example, when increased traffic in the network affects the client-reported quality of delivery, SAS switches to a lower quality state. This results in a reduction in the quantity of data sent, thus helping to improve the situation. This is performed because research has shown [28] that viewers prefer a controlled reduction in multimedia quality to the effect of random losses on the streamed multimedia data. In improved delivery conditions, the QOAS server com- 
ponent gradually increases the quality of the transmitted stream and therefore the transmission rate. In the absence of loss this causes an increase in end-user perceived quality.

\subsection{Quality of Delivery Grading Scheme (QoDGS)}

QoDGS maps some transmission related parameters values and variations and estimates of end-user perceived quality into application-level scores that describe the quality of delivery. It monitors some parameters such as delay, jitter and loss rate, computes estimates of end-user perceived quality using $\mathrm{Q}$ and analyses their shortterm and long-term variations. Short-term monitoring is important for learning quickly about transient effects, such as sudden traffic changes, and for quickly reacting to them. The long-term variations are monitored in order to track slow changes in the overall delivery environment, such as new users in the system. These short-term and long-term periods are set to be an order and two orders of magnitude (respectively) greater than the feedback-reporting interval in the experiments described here.

In the first of QoDGS's three stages, instantaneous values of the monitored parameters are saved in different length sliding windows and their short-term and longterm variations are assessed. At the same time, session-specific lower and higher limits are maintained for each parameter, allowing for corresponding partial scores to be computed in comparison with them. In the second stage, the relative importance of all the monitored parameters in this delivery infrastructure is considered (by weighting their contributions) and the partial scores are used to compute short-term $\left(\mathrm{QoD}_{\mathrm{ST}}\right)$ and long-term $\left(\mathrm{QoD}_{\mathrm{LT}}\right)$ quality of delivery grades. This second stage also takes into account estimates for short-term and long-term end-user perceived quality. In the third stage, $\mathrm{QoD}_{\mathrm{ST}}$ and $\mathrm{QoD}_{\mathrm{LT}}$ are weighted to account for their relative importance and the overall client score $\left(\mathrm{QoD}_{\text {Score }}\right)$ is computed.

Extensive tests were performed in order to make sure that the design of QODGS ensures that best results will be obtained in terms of adaptiveness, responsiveness to traffic variations, stability, link utilization, and end-user perceived quality in local broadband IP-networks. A detailed presentation of QoDGS is given in [4].

\subsection{Server Arbitration Scheme (SAS)}

SAS takes adaptive decisions based on the values of a number of recent feedback reports, in order to minimise the effect of noise in the $\mathrm{QoD}_{\text {scores }}$. This arbitration process is asymmetric, requiring fewer feedback reports to trigger a decrease in quality than for a quality increase. This ensures a fast reaction during bad delivery conditions, helping to eliminate their cause and allowing the network conditions to improve before any quality upgrade. These adaptive decisions are taken to maintain system stability by minimising the number of quality variations. The late arrival of a number of feedback messages is considered as an indication of network congestion, and triggers quality degradations. This permits the streaming scheme to work even if feedback is not available. More details about SAS are presented in [4]. 


\section{Testing Results}

In order to test QOAS performance when delivering multimedia clips in local multiservice broadband IP-networks to home residences and business premises, QOAS was implemented by both a simulation model, built using Network Simulator 2 (NS2) [29], and a prototype system, built using Microsoft Visual C++ 6.0. The simulation model was used for objective testing whereas the prototype system was used for subjective assessment of the end-users' perceived quality.

\subsection{Objective Testing of QOAS}

The objective testing employs NS-2 simulations in order to assess the QOAS performance. The simulation setup requires a network topology, simulation models, multimedia clips, simulation scenarios and performance assessment principles. These issues and the simulation results are presented next.

Network Topology. The NS-2 simulations use a "Dumbbell" topology that assumes a single shared bottleneck link with characteristics as in Figure 2. The $100 \mathrm{~ms}$ latency was chosen such the adaptation of the feedback-based schemes in highly loaded delivery conditions is tested. The sources of traffic, including QOAS server application instances and a source of multimedia-like background traffic are located on one side of the bottleneck link, and the receivers are on the other side. The links are provisioned such as the only significant delays and packet drops are caused by congestion that occurs on the bottleneck.

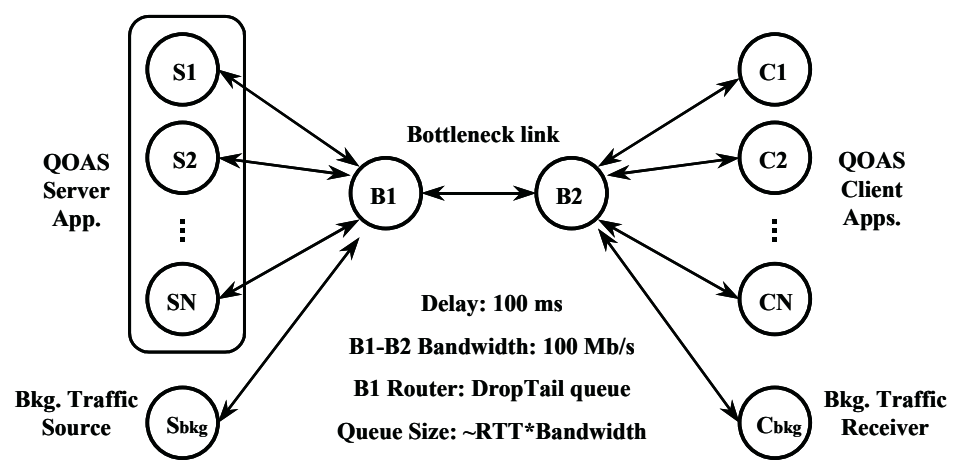

Fig. 2. "Dumbell" topology for NS-2 simulation tests.

Simulation Models. For testing QOAS a simulation model that implements the mechanism described in section III was built, using a five-quality state model for the server. The SAS upgrade period was $6 \mathrm{~s}$ and the downgrade one was $1 \mathrm{~s}$. The QoDGS short-term and long-term periods were set to $1 \mathrm{~s}$ and $10 \mathrm{~s}$, respectively.

When comparing QOAS to other adaptive schemes, NS-2 models for TFRCP [13] and LDA+ [16] were used and maximum rate of $4 \mathrm{Mb} / \mathrm{s}$ was imposed for consistency. 
TFRCP uses estimates of round-trip delay and loss rates to determine the adaptive policy. When loss occurs, the rate of transmission is limited to the one computed by the TCP model [14]. In case of no loss, the current rate is doubled. This TFRCP model uses $5 \mathrm{~s}$ for rate update intervals, as suggested in [13] for latencies greater than $0.1 \mathrm{~s}$, as in this setup.

LDA+ is an AIMD algorithm based on estimates of network condition and bandwidth share used. In zero loss periods, the sender increases its rate with minimum from an estimated bandwidth share rate increase, a bottleneck bandwidth share rate limit, and a corresponding TCP rate update. In nonzero loss periods, the server reduces its rate by a value that depends on the current rate and the rate determined by the TCP model [14]. The LDA+ implementation used an RTCP feedback interval of 5 $\mathrm{s}$ as suggested in [16].

Multimedia Clips. Five video sequences were selected from movies with various types and different degrees of motion content: diehardl - high, jurassic 3 and dontsayaword - average, familyman - low, whereas roadtoeldorado is a typical cartoon sequence. The clips were MPEG-2 encoded at five rates between $2 \mathrm{Mb} / \mathrm{s}$ and $4 \mathrm{Mb} / \mathrm{s}$ using the same frame rate $(25$ frames/sec) and the same IBBP frame pattern ( 9 frames/GOP). Traces were collected, associated with QOAS server states and used during simulations. Statistics related to the ratio between the peak and mean rates for each version of the multimedia sequences used during simulations are presented in Table 1. Peak/mean rate ratios are close related to both the motion complexity and type of multimedia sequences and are the cause for the burstiness of transmissions.

Simulation Scenarios and Results. Simulations involved streaming each multimedia clip indicated in Table 1 for 500s, but 50s long transitory periods at the beginning and the end were not considered when analysing the results. Since in local multi-service broadband IP networks multimedia is expected to account for the majority of traffic, the complex multimedia-like background traffic presented in Figure 3 is used. This traffic simulates possible user interactions such as consecutive play commands that increase the traffic in a staircase-up manner, different frequency pause-play interactions applied on different rate clips and consecutive stop's. In order to create highly loaded network conditions, CBR-UDP background traffic with a rate of $95.5 \mathrm{Mb} / \mathrm{s}$ is generated using NS-2. This traffic represents the well-multiplexed aggregation of a high number of data flows of different types commonly expected in IP networks.

Table 1. Peak/mean rate ratio for all quality versions of the clips used during simulations.

\begin{tabular}{cccccc}
\hline $\begin{array}{c}\text { Quality Version } \\
\text { (average rate) } \\
\text { Clip Name }\end{array}$ & $\begin{array}{c}\mathbf{2 . 0} \mathbf{M b} / \mathbf{s} \\
\text { version }\end{array}$ & $\begin{array}{c}\mathbf{2 . 5} \mathbf{M b} / \mathbf{s} \\
\text { version }\end{array}$ & $\begin{array}{c}\mathbf{3 . 0} \mathbf{M b} / \mathbf{s} \\
\text { version }\end{array}$ & $\begin{array}{c}\mathbf{3 . 5} \mathbf{M b} / \mathbf{s} \\
\text { version }\end{array}$ & $\begin{array}{c}\mathbf{4 . 0 ~ M b / s} \\
\text { version }\end{array}$ \\
\hline diehard1 & 7.48 & 7.43 & 6.31 & 5.65 & 4.06 \\
roadtoeldorado & 6.91 & 6.51 & 6.23 & 6.12 & 6.05 \\
dontsayaword & 5.56 & 4.51 & 4.36 & 4.08 & 3.56 \\
jurassic3 & 4.83 & 4.38 & 4.04 & 3.71 & 3.41 \\
familyman & 3.99 & 3.67 & 3.42 & 3.09 & 2.93 \\
\hline
\end{tabular}




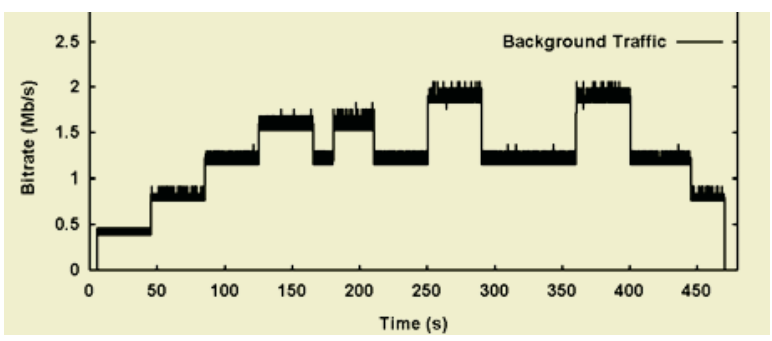

Fig. 3. Multimedia-like background traffic on top of $95.5 \mathrm{Mb} / \mathrm{s}$ CBR traffic.

Table 2. Performance comparison when streaming diehardl with QOAS, TFRCP and LDA+.

\begin{tabular}{ccccc}
\hline $\begin{array}{c}\text { Streaming } \\
\text { Scheme }\end{array}$ & $\begin{array}{c}\text { Avg. Tx. Rate } \\
(\mathbf{M b} / \mathbf{s})\end{array}$ & $\begin{array}{c}\text { Avg. Loss } \\
(\mathbf{\%})\end{array}$ & $\begin{array}{c}\text { Avg. Quality } \\
(\mathbf{1 - 5})\end{array}$ & $\begin{array}{c}\text { Avg. Utilis. } \\
(\boldsymbol{\%})\end{array}$ \\
\hline QOAS & 3.21 & 0.013 & 4.42 & 99.91 \\
TFRCP & 3.16 & 1.057 & 3.79 & 99.88 \\
LDA+ & 2.95 & 1.465 & 3.77 & 99.67 \\
\hline
\end{tabular}

Table 2 presents comparative performance statistics gathered when streaming diehardl using QOAS, TFRCP and LDA+, respectively in these traffic conditions. The performance was assessed in terms of average bit-rate, end-user perceived quality, loss rate and infrastructure utilization. End-user quality is computed using the noreference metric Q [28] and is expressed on the ITU-T five-point scale [30].

Since QOAS maintains very low loss rate $(0.01 \%)$ by successfully adapting even to most difficult background traffic variations, the consequent average end-user perceived quality is between "good" and "excellent" quality level (4.42). Higher loss rates than $1 \%$ are experienced when using both TFRCP and LDA+, determining decreases of the end-user perceived quality much below the "good" perceptual level.

Tests were performed using multimedia clips with different motion content and the results were similar. For exemplification Figure 4 presents a comparison between end-user perceived quality variations when streaming a single multimedia sequence with very complex motion content - diehardl using QOAS, TFRCP and LDA+. QOAS successfully adapts to the staircase-up increase in the background traffic that exceeds the available bandwidth, reducing the quantity of data transmitted and avoiding losses that significantly degrade end-user perceived quality in the TFRCP and LDA+ cases.

When the background traffic varies in a periodic manner with steps comparable to the adaptation step of $0.5 \mathrm{Mb} / \mathrm{s}$ (see Table 1), QOAS obtains better results in terms of perceived quality in comparison to both other solutions due to its conservative policy of slowly increasing the transmission rate to a level determined according to longterm information it maintains. Both LDA+ and TFRCP use a more aggressive manner of recovery after network problems and increase their transmission rate faster. This policy achieves in generally high throughput, but when the background traffic varies sharply like in this situation, it leads to packet loss. 

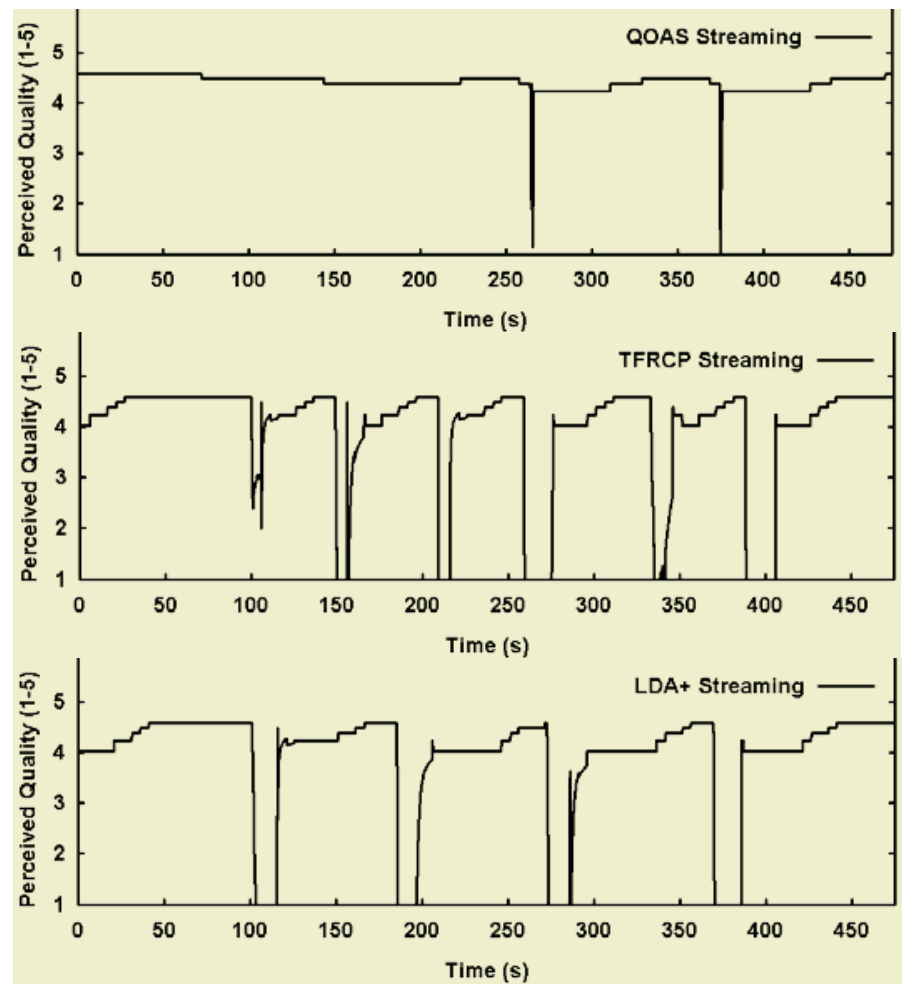

Fig. 4. Comparison between end-user perceived quality when streaming diehardl with QOAS, TFRCP and LDA+.

The effect of a steep increase in the background traffic when the system is already heavily loaded is tested at 250s and 360s. QOAS performs significantly better that both TFRCP and LDA+-based adaptations, reacting much faster to the sharp change in traffic. This minimizes the losses and therefore reduces the period when the perceived quality is degraded from 20 s in TFRCP case and $17 \mathrm{~s}$ in LDA+ case to only $1.2 \mathrm{~s}$.

At the end, the effect of stopping the multimedia cross traffic was tested. All the adaptive schemes increased their rates to compensate for the decrease in background traffic, but TFRCP and LDA+ did this faster than QOAS. However, the difference in the perceived quality between the consequent results was less than $2 \%$.

After obtaining similar results when the other multimedia clips were used for streaming, it can be concluded that the QOAS-based solution showed superior performance to both TFRCP and LDA+. QOAS reacts quickly to changes in network traffic, reducing the quantity of the transmitted data, both preventing and minimizing losses, if they occur. Therefore the consequent end-user perceived quality was much higher than in the other cases when it even reached the "very annoying" level for long periods. QOAS's more conservative upgrade approach pays off if unexpected delivery problems occur. In terms of average utilization, all the solutions have highly performed, although QOAS slightly out-performs the other schemes. 


\subsection{Subjective Testing of QOAS}

Subjective tests were performed in order to verify the objective end-user quality results obtained during simulations. They have involved the prototype system and $60 \mathrm{~s}$ long multimedia sequences taken from movies with different motion content (see Table 1). Increased traffic conditions were emulated using the NistNet network emulator [31] determining QOAS-based adaptations and consequent variations in the viewers' perceived quality.

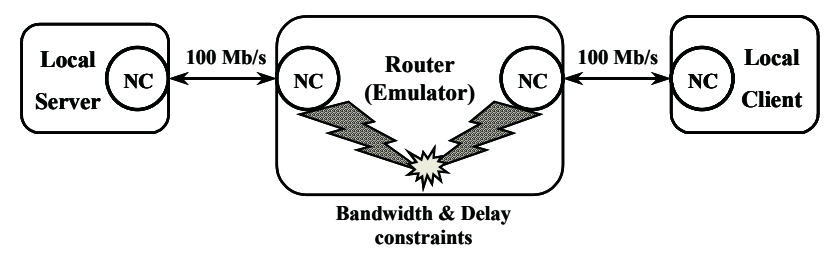

Fig. 5. Testbed setup for subjective testing.

The testbed presented in Figure 5 was set up, testing conditions suggested in [30] were ensured and the Single Stimulus Method with explicit reference was selected as testing methodology for two perceptual tests. These aimed at testing the subjects' perceived quality when using QOAS for streaming in very difficult delivery conditions, as shown by the simulations. The effects of consecutive play commands in the delivery system that are emulated by background traffic that varies in a staircase-up manner are tested in Testl. The effects of periodic variation of traffic with steps of $0.7 \mathrm{Mb} / \mathrm{s}$, higher than the adaptation step of $0.5 \mathrm{Mb} / \mathrm{s}$, are assessed in Test 2 . Figure 6 and Figure 7 show both the background traffic variations and the consequent QOAS rate adaptations during these tests, when the diehardl clip was selected for streaming. Similar results were obtained when the other clips were used.

In each of the two tests 42 subjects, aged between 18 and 48, graded the quality of each streamed clip on the 1-5 ITU-T R. P.910 scale [30]. Among the subjects, 19 and 16 in the first and the second tests respectively wore glasses or contact lenses and none had other visual impairments that may affect their perception of multimedia quality. From the subjects, 23 and 21 respectively were familiar with multimedia streaming, 1 and 2 respectively have considered themselves experts.

The results presented in Table 3 show how QOAS streaming was very appreciated by the test subjects, scoring on average above 4 , the "good" quality level on the ITU$\mathrm{T}$ scale, for all the movies and close to the "good" level for the cartoons sequence.

Table 3. Subjective Test Results: mean end-user perceived quality scores for TEST1 and TEST2.

\begin{tabular}{cccc}
\hline Sequence & Motion Content / Type & Test 1 & Test 2 \\
\hline diehard1 & High / Movie & 4.00 & 4.22 \\
dontsayaword & Average / Movie & 4.18 & 3.98 \\
familyman & Low / Movie & 4.21 & 4.24 \\
roadtoeldorado & Average / Cartoons & 3.74 & 3.85 \\
\hline
\end{tabular}




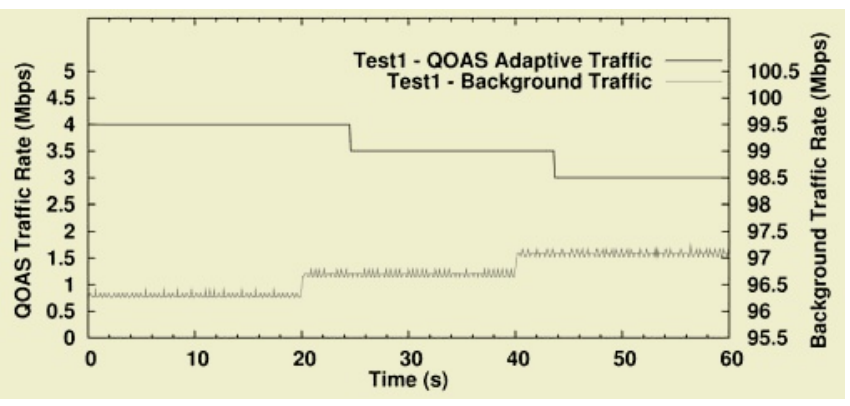

Fig. 6. Test 1: QOAS bit-rate adaptation with background traffic variation when streaming diehardl.

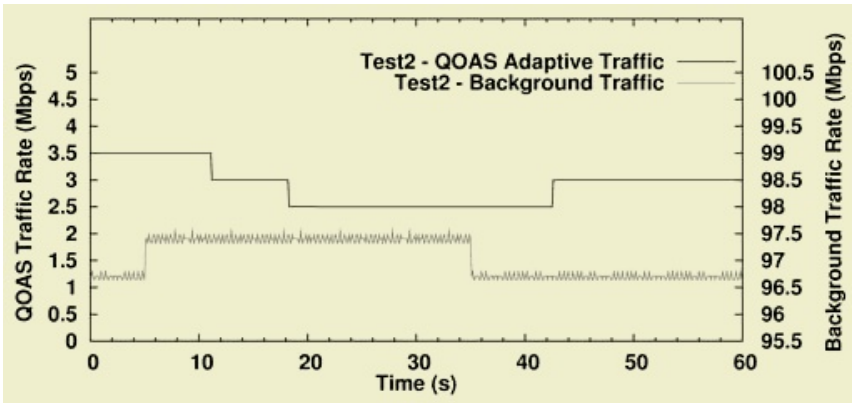

Fig. 7. Test 2: QOAS bit-rate adaptation with background traffic variation when streaming diehardl.

The results of Test1 suggest that the higher the motion complexity of a sequence the lower the subjective appreciation in loaded delivery conditions is, fact supported by an ANOVA test which indicated that the results are significantly different $(\mathrm{p}<$ 0.05). However, during Test 2 when the delivery conditions have triggered loss, the viewers' perceived quality was affected independent from the motion content as shown in Table 3. This finding was supported by an ANOVA test that found the results significantly different $(\mathrm{p}<0.05)$.

Although the results of the second set of subjective test seem higher than those of the first set of tests, by performing t-tests on Test 1 and Test 2 results for each multimedia sequence involved in testing, the null hypothesis that there is no statistical difference between the results of Test 1 and Test 2 respectively cannot be rejected. This finding is stated with a very high level of confidence of $99 \%$ (significance level $\alpha=$ $0.01)$.

At the same time there is a significant statistical difference between the subjective scores obtained for the clips that contain movie scenes and the cartoons clip. This result was confirmed by paired t-tests that were performed for each movie sequence and the cartoons sequence with a significance level of $\alpha=0.01$. A potential cause might be the different MPEG-2 encoding output for the cartoons sequences as shown in Table 1. Unlike for the movie content, for cartoons content the peak/mean ratio computed in relation to the size of the encoded frames does not significantly increase 
with the decrease in the average encoding bit-rate. Also the content with many colors and edges might be more affected in terms of the end-user subjective quality corrupted during streaming.

In conclusion, although slightly lower than the simulation test results obtained in the same conditions (for example when streaming the diehardl sequence the mean scores were 4.42 and 4.22 respectively) the subjective test results verify them and confirm the very good performance of QOAS.

\section{Performance Analysis}

The significant advantages of a QOAS-based solution come with a cost in terms of extra processing requirements and some bandwidth used for feedback.

The fact that this processing is distributed among the QOAS clients whose QoDGSs monitor and grade the quality of streaming at the receivers, significantly reduces the load of the QOAS server machine that runs only the SAS. The QOAS server has only to acquire the client transmitted $\mathrm{QoD}_{\text {Scores }}$, to process them (this can be performed incrementally) and to take adaptive decisions (this does not involve excessive CPU load).

Regarding the feedback, it is significant to mention that each feedback report consists only of a $\mathrm{QoD}_{\text {score }}$. If RTCP packets are used, for standard values for the headers' sizes (20 Bytes - IP header, 8 Bytes - UDP header, 8 Bytes - RTCP receiver report packet header) and for a 4-Byte payload, the feedback packet size becomes 40 Bytes long. For a very low inter-feedback transmission time of $0.1 \mathrm{sec}$ the bandwidth used by feedback for a single client becomes $\mathrm{BW}_{\text {feedback }}=400$ Bytes/s. Since QOAS was designed for local broadband multi-service IP-networks, this represents an insignificant bandwidth usage. For example over 300 customers that are served simultaneously via a gigabit Ethernet infrastructure consume only $0.1 \%$ of the available bandwidth for feedback.

\section{Conclusions and Further Work}

The Quality-Oriented Adaptation Scheme (QOAS) is an end-to-end application-level solution for streaming multimedia that considers the end-user perceived quality as an active factor in the adaptation process. The scheme is tested in conditions expected for delivering multimedia-based services to residential homes or businesses premises via a local broadband multi-service IP network.

Simulation-based objective tests have shown very good performance of QOAS, assessed in terms of remote user perceived quality, average loss rate and network infrastructure utilization when streaming multimedia in loaded network conditions and in the presence of highly variable multimedia-like background traffic. The perceived quality was between the "good" and "excellent" ITU-T quality levels, the loss rate was around $0.01 \%$ and the utilization greater than $99.9 \%$, results that out-perform those obtained when other adaptive schemes such as TFRCP and LDA+ were tested in the same conditions. Subjective tests performed in difficult emulated traffic conditions verify these results. 
These results highly recommend QOAS as a very efficient solution for delivering good quality multimedia-based services in local broadband IP-network to customers, even in increased and highly variable traffic conditions.

Further work will test in detail the performance of QOAS if deployed in local broadband multi-service IP networks against different types of individual traffic flows such as long-lived or short-lived TCP. These tests will study not only the effect this traffic has on multimedia streams transmitted using QOAS, but also the effect QOAS streaming has on the other traffic. In this context QOAS's degree of TCP friendliness is of significant importance. Also experiments that involve streaming of more than one type of multimedia clips at the same time are envisaged. Next QOAS will be extended for multicast transmissions, taking into account some multicast specific characteristics such as multiple feedback and arbitration of heterogeneous client reports in order to make more efficient live multimedia streaming.

\section{Acknowledgements}

The support of the Research Innovation Fund and Informatics Research Initiative of Enterprise Ireland is gratefully acknowledged.

\section{References}

1. Barnett, S. A., Anido, G. J.: A Cost Comparison of Distributed and Centralized Approaches to Video-on-Demand, IEEE Journal Sel. Areas in Comm., vol. 14, no. 6, (1996), 1173-1183

2. Muntean, G.-M., Murphy, L.: An Adaptive Mechanism For Pre-recorded Multimedia Streaming Based On Traffic Conditions, W3C WWW Conf., Honolulu, HI, USA, (2002)

3. Muntean, G.-M., Murphy, L.: Adaptive Pre-recorded Multimedia Streaming, IEEE GLOBECOM, Taipei, Taiwan, (2002)

4. Muntean, G.-M., Perry, P., Murphy, L.: A New Adaptive Multimedia Streaming System for All-IP Multi-Service Networks, IEEE Trans. on Broadcasting, vol. 50, no. 1, (2004), 110

5. Muntean, G.-M., Perry, P., Murphy, L.: Performance Comparison of Local Area Video Streaming Systems, IEEE Communication Letters, vol. 8, no. 5, (2004), 326-328

6. Muntean, G.-M., Perry, P., Murphy, L.: A Quality-Orientated Adaptation Scheme for Video-on-Demand, IEE Electronic Letters, vol. 39, no. 23, (2003), 1689-1690

7. Dravida, S., Gupta, D., Nanda, S., Rege, K., Strombosky, J., Tandon, M.: Broadband Access over Cable for Next-Generation Services: A Distributed Switch Architecture, IEEE Comm. Magazine, vol. 40, no. 8, (2002), 116-124

8. Wang, X, Schulzrinne, H.: Comparison of Adaptive Internet Multimedia Applications, IEICE Trans. on Comm., vol. E82-B/6, (1999), 806 - 818

9. Wu, D., Hou, Y. T., Zhu, W., Zhang, Y.-Q., Peha, J. M.: Streaming Video over the Internet: Approaches and Directions, IEEE Trans. on Circuits and Systems for Video Technology, vol. 11, no. 3, (2001), 282-300

10. Wu, D., Hou, Y. T., Zhang, Y.-Q.: Transporting Real-time Video over the Internet: Challenges and Approaches, Proc. IEEE, vol. 88, no. 12, (2000) 
11. Kanakia, H., Mishra, P., Reibman, A.: An Adaptive Congestion Control Scheme for Realtime Packet Video Transport, Proc. ACM SIGCOMM, San Francisco, USA, (1993), 20-31

12. Bolot, J.-C., Turletti, T.: A Rate Control Mechanism for Packet Video in the Internet, Proc. IEEE INFOCOM, Toronto, Canada, (1994), 1216-1223

13. Padhye, J., Kurose, J., Towsley, D., Koodli, R.: A Model Based TCP Friendly Rate Control Protocol, Proc. ACM NOSSDAV, New Jersey, (1999)

14. Padhye, J., Firoiu, V., Towsley, D., Kurose, J.: Modeling TCP Throughput: A Simple Model and its Empirical Validation, Proc. ACM SIGCOMM, Vancouver, Canada, (1998)

15. Sisalem, D., Schulzrinne, H.: The Loss-Delay Adjustment Algorithm: A TCP-friendly Adaptation Scheme, Proc. ACM NOSSDAV, UK, (1998)

16. Sisalem, D., Wolisz, A.: LDA+ TCP-Friendly Adaptation: A Measurement and Comparison Study, Proc. ACM NOSSDAV, USA, (2000)

17. Rejaie, R., Handley, M., Estrin, D.: RAP: An End-to-end Rate-based Congestion Control Mechanism for Realtime Streams in the Internet, Proc. IEEE INFOCOM, New York, NY, USA, (1999), 1337-1345

18. Rejaie, R., Handley, M., Estrin, D.: Layered Quality Adaptation for Internet Video Streaming, IEEE J. Sel. Areas of Comm., vol. 18, no. 12, (2000), 2530-2543

19. Jacobs, S., Eleftheriadis, A.: Streaming Video Using Dynamic Rate Shaping and TCP Congestion Control, Journal of Visual Comm. and Image Repres., vol. 9, no. 3, (1998), 221-222

20. McCanne, S., Jacobson, V., Vetterli, M.: Receiver-Driven Layered Multicast, Proc. ACM SIGCOMM, Stanford, USA, (1996), 117-130

21. Vicisano, L., Crowcroft, J., Rizzo, L.: TCP-like Congestion Control for Layered Multicast Data Transfer, Proc. IEEE INFOCOM, vol. 3, (1998), 996-1003

22. Rhee, I., Ozdemir, V., Yi, Y.: TEAR: TCP Emulation at Receivers - Flow Control for Multimedia Streaming, Technical Report, CS Department, NCSU, (2000)

23. Yeadon, N., García, F., Hutchison, D., Shepherd, D.: Filters: QoS Support Mechanisms for Multipeer Communications, IEEE J. Sel. Areas in Comm., vol. 14, no. 7, (1996), 12451262

24. Wang, L., Luthra, A., Eifrig, B.: Rate Control for MPEG Transcoders, IEEE Trans. on Circuits and Systems for Video Technology, vol. 11, no. 2, (2001)

25. RealNetworks, SureStream, [Online]. Available: http://www.realnetworks.com

26. Microsoft, Windows Media, MBR, [Online]. Available: http://www.microsoft.com

27. Ghinea, G., Thomas, J. P., QoS Impact on User Perception and Understanding of Multimedia Video Clips, Proc. ACM Multimedia, Bristol, UK, (1998)

28. Verscheure, O., Frossard, P., Hamdi, M.: User-Oriented QoS Analysis in MPEG-2 Video Delivery, Journal of Real-Time Imaging, vol. 5, no. 5, (1999), 305-314

29. Network Simulator-2, [Online]. Available: http://www.isi.edu/nsnam/ns/

30. ITU-T Recommendation P.910: Subjective Video Quality Assessment Methods for Multimedia Applications, (1999)

31. NIST Net, [Online]. Available: http://snad.ncsl.nist.gov/itg/nistnet 